\title{
The optical counterpart of the ultraluminous X-ray source NGC6946 ULX-1
}

\author{
P. K. Abolmasov, S. N. Fabrika and O. N. Sholukhova \\ Special Astrophysical Observatory of the Russian AS, Nizhnij Arkhyz 369167, Russia \\ email: pasha@sao.ru
}

\begin{abstract}
We present a study of a peculiar nebula MF16 associated with an Ultraluminous X-ray Source NGC6946 ULX-1. We use integral-field and long-slit spectral data obtained with the 6-m telescope (Russia). The nebula was for a long time considered powered by strong shocks enhancing both high-excitation and low-excitation lines. However, kinematical properties point to rather moderate expansion rates $\left(V_{S} \sim 100 \div 200 \mathrm{~km} \mathrm{~s}^{-1}\right)$. The total power of the emissionline source exceeds by one or two orders of magnitude the power observed expansion rate can provide, that points towards the existence of an additional source of excitation and ionization. Using CLOUDY96.01 photoionization code we derive the properties of the photoionizing source. Its total UV/EUV luminosity must be about $10^{40} \mathrm{erg} / \mathrm{s}$.
\end{abstract}

Keywords. X-rays: individual (NGC6946 X8) - ISM: individual (MF16) - ISM: jets and outflows - ultraviolet: general

\section{Introduction}

Quite a large number of Ultraluminous X-ray Sources (ULXs) are associated with emission-line nebulae (ULX Nebulae, ULXNe), mostly large-scale bubbles powered by shock waves (Pakull \& Mirioni 2003). However, several exceptions are known like the nebula associated with HoII X-1 (Lehmann et al. 2005), that is clearly a photoionized $\mathrm{H}$ II region. Another well-known example is the nebula MF16 coincident with the ULX NGC6946 ULX1.

The attention to MF16 was first drawn by Blair \& Fesen (1994), who identified the object as a Supernova Remnant (SNR), according to the emission-line spectrum with bright collisionally-excited lines. It was long considered an unusually luminous SNR, because of its huge optical emission-line, $L_{H \alpha}=1.9 \times 10^{39} \mathrm{erg} \mathrm{s}^{-1}$, according to Blair \& Fesen (1994), for the tangential size $20 \times 34 \mathrm{pc}-$ and in X-rays, $L_{X}=2.5 \times 10^{39} \mathrm{erg} \mathrm{s}^{-1}$ in the $0.5-8 \mathrm{keV}$ range, according to the luminosities given by Roberts \& Colbert (2003).

However, it was shown by Roberts \& Colbert (2003), that the spectral, spatial and timing properties of the X-ray source do not agree with the suggestion of a bright SNR, but rather suppose a point source with a typical "ULX-like" X-ray spectrum: cool Multicolor Disk (MCD) and a Power Law (PL) component. So, apart from the physical nature of the object, MF16 should be considered a $U L X$ nebula, one of a new class of objects, described by Pakull \& Mirioni (2003).

\section{Optical Spectroscopy}

All the data were obtained on the SAO $6 \mathrm{~m}$ telescope, Russia. Two spectrographs were used: panoramic MultiPupil Fiber Spectrograph MPFS (Afanasiev et al. 2001) and SCORPIO focal reducer (Afanasiev \& Moiseev 2005) in long-slit mode. The details of data reduction processes and analysis technique will be presented in Abolmasov et al. (2007). 
Panoramic spectroscopy has the advantage of providing unbiased flux estimates. However, SCORPIO results have much higher signal-to-noise ratio and reveal rich emission-line spectrum of [Fe III]. We also confirm the estimates of the total nebula emission-line luminosities by Blair et al. (2001). $H \beta$ line luminosity obtained from our MPFS data is $L(H \beta)=(7.2 \pm 0.2) \times 10^{37} \mathrm{erg} \mathrm{s}^{-1}$.

Using line ratios for the integral spectrum we estimate the mean parameters of emitting gas as: $n_{e} \simeq 500 \pm 100 \mathrm{~cm}^{-3}, T_{e} \simeq(1.9 \pm 0.2) \times 10^{4} \mathrm{~K}$. Interstellar absorption is estimated as $A_{V} \sim 1^{\mathrm{m}} \cdot 3$, close to the Galactic value $\left(A_{V}^{\text {Gal }}=1^{\mathrm{m}} \cdot 14\right.$, according to Schlegel et al. (1998)).

We confirm the estimate of the expansion rate obtained by Dunne et al. (2000), coming to the conclusion that the expansion velocity is $V_{S} \lesssim 200 \mathrm{~km} \mathrm{~s}^{-1}$. In this case the total emission-line luminosity can be estimated using for example the equations by Dopita \& Sutherland (1996):

$$
\begin{aligned}
& F_{H \beta}=7.44 \times 10^{-6}\left(\frac{V_{s}}{100 \mathrm{~km} \mathrm{~s}^{-1}}\right)^{2.41} \times\left(\frac{n_{2}}{\mathrm{~cm}^{-3}}\right)+
\end{aligned}
$$

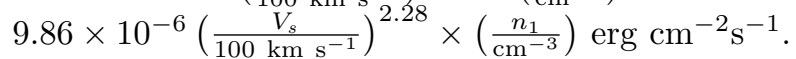

Here $V_{S}$ is the shock velocity and $n_{1}$ the pre-shock hydrogen density. If the surface area is known, one can obtain the total luminosity in $H \beta$ from here. For $V_{S}=200 \mathrm{~km} / \mathrm{s}$ and $n_{1}=1 \mathrm{~cm}^{-3}$ it appears to be $L(H \beta) \simeq 1.6 \times 10^{36} \mathrm{erg} \mathrm{s}^{-1}$, that is too low compared to the observed value. So we suggest an additional source of power providing most of the energy of the optical nebula.

\section{Photoionization Modelling}

We have computed a grid of CLOUDY96.01 (Ferland et al. 1998) photoionization models in order to fit MF16 spectrum avoiding shock waves. We have fixed X-ray spectrum known from Chandra observations (Roberts \& Colbert 2003), assuming all the plasma is situated at $10 \mathrm{pc}$ from the central point source, and introduced a blackbody source with the temperature changing from $10^{3}$ to $10^{6} \mathrm{~K}$ and integral flux densities from 0.01 to 100 erg $\mathrm{cm}^{-2} \mathrm{~s}^{-1}$.

The best fit parameters are $\lg T(K)=5.15 \pm 0.05, F=0.6 \pm 0.1 \mathrm{erg} \mathrm{cm}^{-2} \mathrm{~s}^{-1}$, that suggests quite a luminous ultraviolet source: $L_{U V}=(7.5 \pm 0.5) \times 10^{39} \mathrm{erg} \mathrm{s}^{-1}$. The UV source is more than 100 times brighter then what can be predicted by extrapolating the thermal component of the best-fit model for X-ray data (Roberts \& Colbert 2003).

\section{Ultraluminous UV sources?}

At least for one source we have indications that its X-ray spectrum extends in the EUV region. It is interesting to analyse the implications in the frameworks of two most popular hypotheses explaining the ULX phenomenon.

For the standard disk of Shakura \& Sunyaev (1973) the inner temperature scales as:

$$
T_{i n} \simeq 1 \mathrm{keV}\left(\frac{M}{M_{\odot}}\right)^{-1 / 4}\left(\frac{\dot{M}}{\dot{M}_{c r}}\right)^{1 / 4} .
$$

In Figure 1 we present the reconstructed Spectral Energy Distribution of NGC6946 ULX1 including optical identification by Blair et al. (2001) and the best-fit blackbody from our model. For comparison, a set of MCD SEDs for IMBHs accreting at $1 \%$ of critical rate is shown. To explain the high EUV luminosity and roughly flat SED in the EUV region, a rather high $\mathrm{IMBH}$ mass is needed, $M \gtrsim 10^{4} M_{\odot}$. 


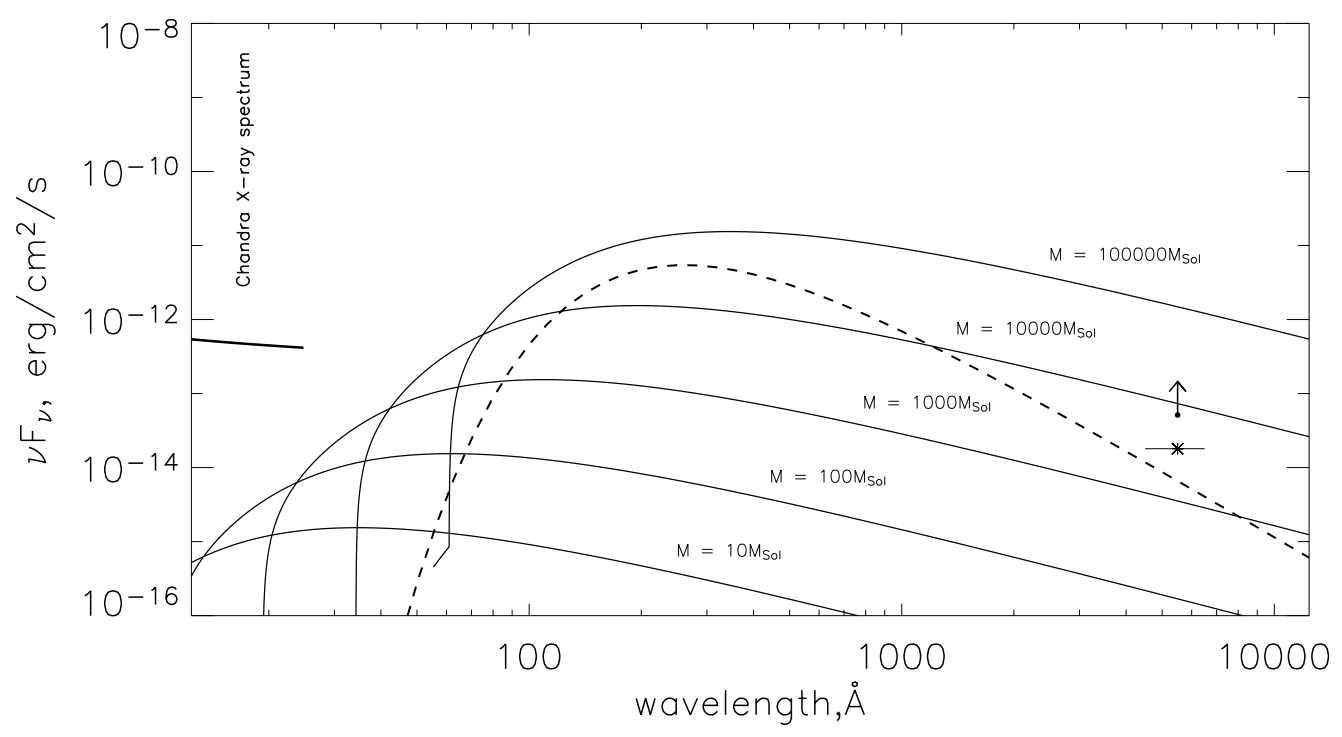

Figure 1. NGC6946 ULX1 SED reconstruction. Optical source $d$ (Blair et al. 2000) is shown by an asterisk, and the upward arrow above indicates the unabsorbed optical luminosity: it is the lower estimate because only Galactic absorption was taken into account, $A_{V}=1^{\mathrm{m}} .14$ according to Schlegel et al. (1998). Dashed line represents the best-fit blackbody from our CLOUDY fitting. Thin solid lines are MCD models for accreting IMBHs with infinite outer disk radii. Mass accretion rate was set everywhere to $0.01 \dot{M}_{c r}$.

For supercritical disk this relation breaks (Poutanen et al. 2006), and the outcoming radiation becomes much softer, except for the X-rays escaping along the disk axis (Fabrika et al. 2007). Most part of the luminosity is supposed to be reprocessed into EUV and UV quanta, creating the nearly-flat SED of NGC6946 ULX1. In optical/UV range contribution of the donor star may become significant.

In Abolmasov et al. (2007) we make estimates for the detectability of ULXs with GALEX, coming to the conclusion that at least some of them (the sources with lower Galactic absorption) may be bright enough targets even for low-resolution spectroscopy.

\section{Conclusions}

We conclude that MF16 is most likely a dense shell illuminated from inside. This can be a certain stage of the evolution of a ULXN, when the central source is bright and the shell itself rather compact. We suggest that ULXs must be luminous EUV sources as well in some cases, and may be also luminous UV sources.

\section{Acknowledgements}

This work was supported by the RFBR grants NN 05-02-19710, 04-02-16349, 06-0216855 .

\section{References}

Abolmasov, P., Fabrika, S., Sholukhova, O. \& Afanasiev, V. 2005, in Science Perspectives for 3D Spectroscopy, ed. M. Kissler-Patig, M. M. Roth. \& J. R. Walsh (Springer Berlin/Heidelberg); astro-ph/0602369

Abolmasov, P., Fabrika, S. \& Sholukhova, O. 2007, in preparation

Afanasiev, V. L., Dodonov, S. N. \& Moiseev, A. V. 2001, in Stellar Dynamics: from Classic to Modern, eds. Osipkov L.P., Nikiforov I.I., Saint Petersburg, 103 
Afanasiev, V. \& Moiseev, A. 2005, Astronomy Letters, 31, 194

Begelman, M. C. 2002, ApJ, 568, L97

Blair, W. P. \& Fesen, R. A. 1994, ApJ, 424, L10371.8

Blair, W. P., Fesen, R. A. \& Schlegel, E. M. 2001, AJ, 121, 1497

Colbert, E. J. M. \& Miller, E. C. 2005, astro-ph/0402677

Dopita, M. A. \& Sutherland, R. S. 1996, ApJSS, 102, 161

Dunne, B. C., Gruendl, R. A. \& Chu, Y.-H. 2000, AJ, 119, 1172

van Dyk, S. D., Sramek, R. A. \& Weiler, K. W. 1994, ApJ, 425, 77

Fabian, A. C. \& Terlevich, R. 1996, MNRAS, 280, L5

Fabrika, S. \& Mescheryakov, A. 2001, In Galaxies and their Constituents at the Highest Angular Resolutions, Proceedings of IAU Symposium No. 205, R. T. Schilizzi (Ed.), p. 268, astro$\mathrm{ph} / 0103070$

Fabrika, S. 2004, Astrophysics and Space Physics Reviews, 12, 1

Fabrika, S., Abolmasov, P. \& Sholukhova, O. 2005, in Science Perspectives for 3D Spectroscopy, eds. Kissler-Patig M., Roth M. M. \& Walsh J. R.

Fabrika, S., Karpov, S. \& Abolmasov, P. 2007, in preparation

Ferland, G. J., Korista, K. T., Verner, D. A., Ferguson, J. W., Kingdon, J. B. \& Verner, E. M. 1998, PASP, 110, 761

King, A. R., Davies, M. B., Ward, M. J., Fabbiano, G. \& Elvis, M. 2001, å, 552, 109

Lehmann, I., Becker, T., Fabrika, S., Roth, M., Miyaji, T., Afanasiev, V. et al. 2005, å, 431, 847

Liu, J.-F. \& Bregman, N. 2005, ApJSS, 157, 59L

Matonick, D. M. \& Fesen, R. A. 1997, ApJSS, 112, 49

Osterbrock, D. E. 1974, Astrophysics of Gaseous Nebulae, San Francisco, eds. W. H. Freeman and Company

Pakull, M. W. \& Mirioni, L. 2003, RevMexAA (Serie de Conferencias), 15, 197

Poutanen, J., Fabrika, S., Butkevich, A. \& Abolmasov, P. 2006, in press

Roberts, T. P. \& Colbert, E. J. M. 2003, MNRAS, 341, 49

Schlegel, D. J., Finkbeiner, P. F. \& Davis, M. 1998, ApJ, 500, 525

Shakura, N. I. \& Sunyaev, R. A. 1973, A\&A, 24, 337

Swartz, A. D., Ghosh, K. K., Tennant, A. F. \& Wu, K. 2004, ApJSS, 154, 519

SuZY Collin: Perhaps there can be a similarity with LINERs, except that in LINERs the black holes are supermassive.

Pavel Abolmasov: Yes indeed, objects discussed in my talk have line ratios resembling LINERs. 